\title{
Intervenção fisioterapêutica no preparo de fortalecimento pélvico para o parto
}

\section{normal}

\author{
Physiotherapeutic intervention in preparing pelvic strengthening for a normal birth \\ Intervención fisioterapéutica en la preparación del fortalecimiento pélvico para un parto normal
}

\author{
Josiany Resplandes da Silva \\ ORCID: https://orcid.org/0000-0001-9154-6997 \\ Instituto Educacional Santa Catarina, Brasil \\ Faculdade Guaraí, Brasil \\ E-mail: josianyresplandes@outlook.com \\ Eliane Candida da Silva \\ ORCID: https://orcid.org/0000-0001-7299-9173 \\ Instituto Educacional Santa Catarina, Brasil \\ Faculdade Guaraí, Brasil \\ E-mail: elianecandida@hotmail.com \\ Karla Camila Correia da Silva \\ ORCID: https://orcid.org/0000-0003-1538-7028 \\ Instituto Educacional Santa Catarina, Brasil \\ Faculdade Guaraí, Brasil \\ E-mail:karlacamilac@yahoo.com.br
}

\begin{abstract}
Resumo
A qualidade da vida da mulher gestante que opta por parto normal leva a considerações na saúde pública e local. Um parto normal leva a alterações na musculatura do assoalho pélvico que pode levar a complicações fisiológicas. Apresentar informações acerca da conduta fisioterapêutica no preparo do fortalecimento pélvico após parto normal consistem nos objetivos centrais deste trabalho. $\mathrm{O}$ estudo foi apresentado na forma de revisão bibliográfica por meio de artigos científicos publicados em revistas cientificas nacionais e internacionais. Foram selecionados trabalhos indexados nas bases de dados ScienceDirecct, PubMed, Medline, Web of Science, EMBASE, LILACS, Scielo e Google Acadêmico. Anatomia humana do assoalho pélvico, suas inervações e contextos foram apresentados neste trabalho, também foi contemplado trabalhos indexados que envolvem a contextualização de parto normal, assoalho pélvico, enfermidades e suas vertentes. Um programa fisioterapêutico nestas situações é de suma importância para qualidade de vida da mulher e associados.
\end{abstract}

Palavras-chave: Assoalho pélvico; Gestantes; Parto normal; Fisioterapêutica.

\begin{abstract}
The quality of life of pregnant women who choose normal childbirth leads to public and local health considerations. A normal birth leads to changes in the pelvic floor muscles that can lead to physiological complications. Presenting information about physical therapy conduct in the preparation of pelvic strengthening after vaginal delivery are the main objectives of this work. The study was presented as a literature review through scientific articles published in national and international scientific journals. Papers indexed in the databases ScienceDirect, PubMed, Medline, Web of Science, EMBASE, LILACS, Scielo and Academic Google were selected. Human anatomy of the pelvic floor, its innervations and contexts were presented in this work, indexed works that involve the contextualization of normal birth, pelvic floor, diseases and their aspects were also contemplated. A physical therapy program in these situations is extremely important for the quality of life of women and associates.
\end{abstract}

Keywords: Pelvic floor; Pregnant women; Vaginal delivery; Physical therapy.

\section{Resumen}

La calidad de vida de las mujeres embarazadas que optan por un parto normal lleva a consideraciones de salud pública y local. Un parto normal provoca cambios en los músculos del suelo pélvico que pueden provocar complicaciones fisiológicas. Presentar información sobre la realización de fisioterapia en la preparación del fortalecimiento pélvico después del parto vaginal son los principales objetivos de este trabajo. El estudio se presentó como una revisión de la literatura a través de artículos científicos publicados en revistas científicas nacionales e internacionales. Se seleccionaron trabajos indexados en las bases de datos ScienceDirecct, PubMed, Medline, Web of Science, EMBASE, LILACS, Scielo y Academic Google. En este trabajo se presentó la anatomía humana del suelo pélvico, sus inervaciones y contextos, también se contemplaron trabajos indexados que involucran la contextualización del parto normal, suelo 
pélvico, enfermedades y sus aspectos. Un programa de fisioterapia en estas situaciones es extremadamente importante para la calidad de vida de las mujeres y sus asociados.

Palabras clave: Suelo pélvico; Gestante; Parto vaginal; Fisioterapia.

\section{Introdução}

A qualidade da saúde da mulher é um caso global de saúde pública mundialmente (Anastasiadi et al., 2017; Permanyer \& Boertien, 2019) onde a gestação mostra-se com vertentes na saúde da mulher e pós o parto ocorrem mudanças corporais que necessitam de atenção médica e fisioterapêutica (Assis, 2010). Há diversas problemáticas de saúde que envolvem o parto natural como fraqueza e dilatação do assoalho pélvico, trazendo inúmeras complicações para a vida da mulher (Andrade, 2021). Nesta situação há uma sobrecarga da musculatura do assoalho pélvico (MAP) e alterações biomecânicas e hormonais normais durante a gestação e entre outros tais como alterações posturais, ganho excessivo de peso, hábitos urinários inadequados podem levar a gestantes a disfunções fisiológicas, físicas e hormonais (Nygaard et al., 2017)

O acompanhamento fisioterapêutico é indicado com umas das primeiras escolhas da Sociedade Internacional de Continência (Castro, Machado \& Trindade, 2019) A fisioterapia dispõe de diversos tipos de tratamentos para IU, que tem como objetivo potencializar o fortalecimento do assoalho pélvico; entre eles se destaca os cones vaginais. O cone vaginal, foi proposto por Plevnik para o fortalecimento resistido e progressivo dos músculos do assoalho da pelve. Essa terapia proporciona uma percepção da região perineal, trazendo assim uma força muscular do assoalho pélvico e o recrutamento de fibras musculares do tipo I e tipo II (Moreno, 2009)

A atuação do fisioterapeuta no fortalecimento e na reeducação perineal do assoalho pélvico é de suma importância e tem como objetivo "melhorar a força de contração das fibras musculares, prevenir disfunções sexuais e incontinências. Com isso, os exercícios de fortalecimento para o assoalho pélvico são eficazes para melhorar a força da musculatura, a sensibilidade, aumentar o fluxo sanguíneo da região perineal, favorecendo a melhora do desejo sexual, orgasmo, da lubrificação, e da excitação diante disso fortalecer o assoalho pélvico previne incontinência urinaria, disfunções sexuais, prolapso genital e melhora a satisfação e a qualidade sexual" (Nagamine et al., 2021).

Com base nestas informações, uma revisão literária mostrando as vertentes relacionadas com a conduta fisioterapêutica no fortalecimento do assoalho pélvico após parto normal é de suma importância para contribuir para o leque de conhecimentos gerais da saúde para a comunidade médica e da fisioterapia.

\section{Metodologia}

Este estudo trata-se de uma revisão bibliográfica e descritiva de abordagem qualitativa. Tendo como base para sua discussão teórica, artigos científicos e demais produções intelectuais (Pereira et al., 2018).

Foram selecionados trabalhos indexados nas bases de dados: ScienceDirect, PubMed, Medline, Web of Science, EMBASE, LILACS, Scielo e Google Acadêmico em um total de 14 artigos selecionados entre o ano de 2000 a 2021. Foram utilizadas as seguintes palavras-chave na língua portuguesa: "Fisioterapêutico" "Fortalecimento pélvico", "Gestantes" e na língua inglesa "Physiotheraphy", "pelvic strengthening ", "Pregnant".

\section{Resultados e Discussão}

\subsection{Músculos do assoalho pélvico}

Denominado como membrana muscular de espessura alterável conhecida como diafragma juntamente com músculos levantadores do ânus (puborretal, pubococcígeo, iliococcígeo e coccígeo), além disso, a pubococcígeo e dividido em puboperineal, puboprostático para homens, mas tambem pubovaginal para mulheres e, puboanal. Na anatomia macroscópica a 
pelve estabelecer como uma importante transição entre a região toracoabdominal e os membros inferiores, tendo a função de deambulação, abriga os sistemas urogenital e digestivo baixo, atuando como um conduto de artérias e veias, vasos linfáticos e nervos que e necessários pro funcionamento do organismo. Por si só, a pelve se constitui como estrutura musculoesquelética composta por ossos do quadril (anca) e sacrococcígeos, juntamente com várias camadas dos músculos. Adicionalmente e dividido em pelve maior (falsa) e pelve menor (verdadeira).

A pelve falsa é uma das áreas amplas prontamente acima do início da entrada, entre as asas ilíacas, enquanto a pelve verdadeira é a área em meio a a entrada e a saída da pelve, possuindo duas paredes laterais, uma parede posterior (ossos sacrococcígeos) e um assoalho muscular. Já a parte inferior da pelve é fechada por um diafragma muscular e uma membrana perineal e são denominados assoalho pélvico, o qual auxilia na prevenção dos conteúdos da cavidade pélvica e manutenção da continência urinária. Os principais músculos estão demonstrados na Figura 1 (Netter, 2000).

Figura 1. Partes musculares anteriores e posteriores do assoalho pélvico.

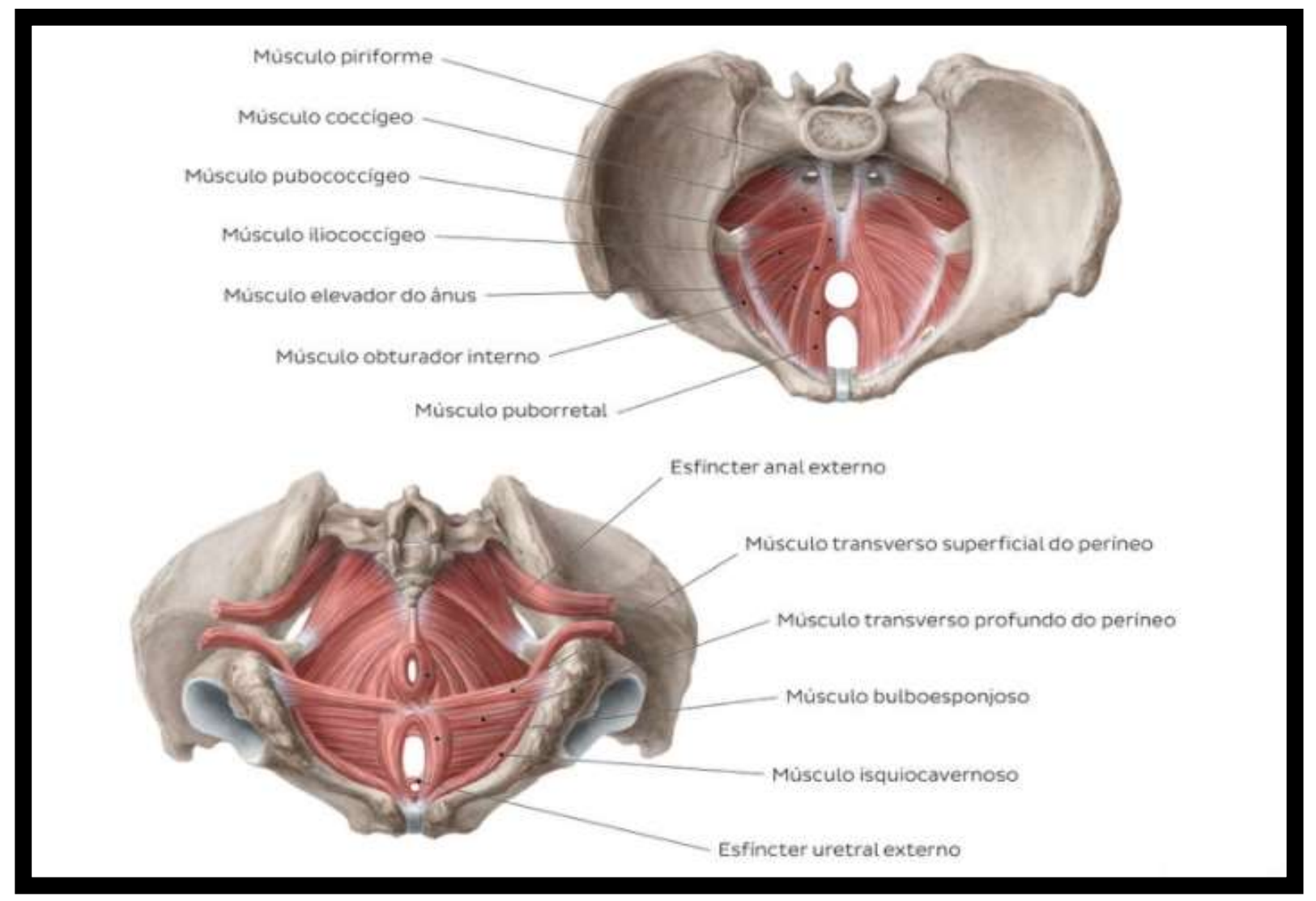

Fonte: https://www.kenhub.com/pt/library/anatomia/musculos-do-assoalho-pelvico.

Já a Tabela 1, resume-se nas especificações do assoalho pélvico demonstrando por meio da anatomia as suas vertentes. 
Tabela 1. Resumo das vertentes musculares do assoalho pélvico.

\begin{tabular}{|l|l|}
\hline Puborretal & $\begin{array}{l}\text { Origem: Superfície posterior dos corpos dos ossos púbicos } \\
\text { Inserção: nenhuma (forma uma alça puborretal posterior ao reto) } \\
\text { Inervação: nervo para o levantador do ânus (S4) }\end{array}$ \\
\hline Pubococcígeo & $\begin{array}{l}\text { Origem: superfície posterior dos corpos dos ossos púbicos (lateral ao puborretal) Inserção: } \\
\text { ligamento anococcígeo, cóccix, corpo perineal e musculatura da próstata/vagina } \\
\text { Inervação: nervo para o levantador do ânus (S4), ramos através dos ramos inferiores } \\
\text { retais/perineais do nervo pudendo (S2-S4) }\end{array}$ \\
\hline Iliococcígeo & $\begin{array}{l}\text { Origem: arco tendíneo da fáscia do obturador interno, espinha isquiática } \\
\text { Inserção: ligamento anococcígeo, cóccix } \\
\text { Inervação: nervo para o levantador do ânus (S4) }\end{array}$ \\
\hline Coccígeo (Isquiococcígeo) & $\begin{array}{l}\text { Origem: espinha isquiática } \\
\text { Inserção: extremidade inferior do sacro, cóccix } \\
\text { Inervação: ramo anterior dos nervos espinhais S4-S5 }\end{array}$ \\
\hline
\end{tabular}

Fonte: Autores.

Já sobre a inervação, as divisões do plexo sacral (Figura 2) contribuem para a inervação de grupo dos músculos do levantador do ânus. "O nervo pudendo, se origina dos segundo ao quarto segmentos sacrais (S4-S4), inerva se abertamente o músculo pubococcígeo. Partes diretas que se originam do quarto segmento sacral que desenvolvem o nervo para o levantador do ânus (S4), além disso inerva o pubococcígeo. Os músculos remanescentes coccígeo e iliococcígeo se dão inervados por ramos diretos do quarto e do quinto segmentos (S4 e S5) do plexo sacral” (Netter, 2000).

Figura 2. Plexo sacral em ramos.

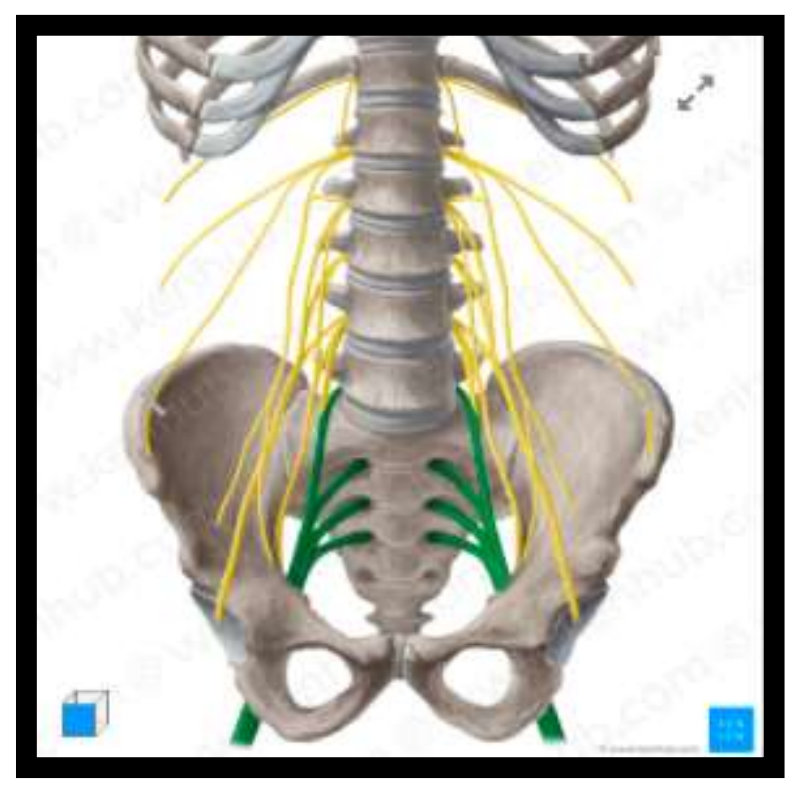

Fonte: Adaptado de Netter (2000).

\subsection{A fisiologia do parto natural e Exercícios fisioterapêuticos no fortalecimento pélvico}

$\mathrm{Na}$ condução do parto natural, existem alguns fatores fisiológicos a serem considerados tais como, a ruptura de membranas corioaminióticas ou a saída do tampão mucoso, o qual indica o início do trabalho de parto mediado por hormônios como a prostaglandina que atua no início do trabalho do parto e juntamente com a progesterona e a relaxina (amolece as 
articulações e ligamentos pélvicos), preparam o colo do útero e a pelve materna para o trabalho de parto. Já a ocitocina, que é um hormônio de suma importância em toda a gravidez, ele atua nas contrações uterinas. Nas contrações uterinas, o trabalho de parto é subdivido, no caso do parto natural, em três fases: Período latente, fase ativa e segunda fase, os quais vão desde o início das dilatações (em torno de $4 \mathrm{~cm}$ ) até a dilatação total e desprendimento do feto (GOODRICH, 1953).

Os principais exercícios para fortalecimento pélvico consistem na sua realização antes e depois do parto, devido a ocorrência de forças exigentes para sustentação do bebê e devido a esta força exigida, há um enfraquecimento da musculatura. Para esta situação, têm determinados exercícios tais como decúbito ventral, decúbito dorsal sentada, posição ortostática, decúbito lateral e cócoras (NAGAMINE ET AL., 2021). A bola suíça é um grande recurso dentro da atividade física para fortalecimento pélvico, ainda mostrada na literatura, principalmente em atividades de Pilates (ULDAHL ET AL., 2021) (Figura 3).

Figura 3. Exercício com bola de ginástica para fortalecimento pélvico.

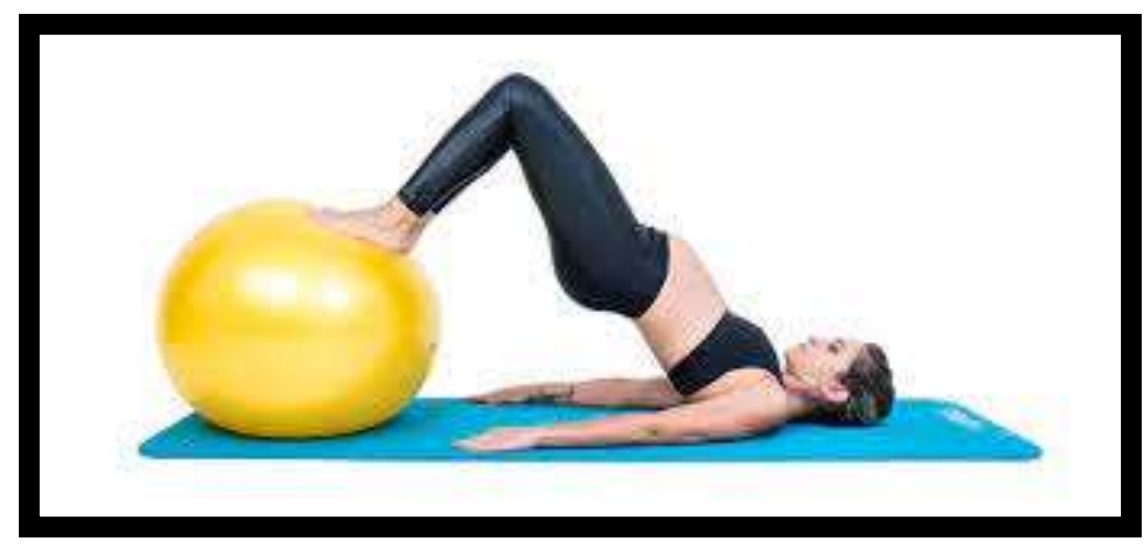

Fonte: https://blogpilates.com.br/exercicios-de-pilates-com-bola-fit-ball/

O treinamento das musculaturas do assoalho pélvico promove a força do músculo esquelético que estão inseridos no corpo cavernoso do clítoris, o qual promove melhor resposta do reflexo sensório-motor como a contração involuntária deste motor, melhorando o fluxo sanguíneo pélvico. A cinesioterapia é consiste em alguns exercícios de contração longa e rápida podendo ser atuada entre três séries de dez repetições. Outro exercício para fortalecimento pélvico destaca-se a contração abdominal associada com tração perineal, além disso, existem os equipamentos específicos para isto, tais como dinamômetro, eletro miógrafo, manômetro ou perineômetro, avaliação funcional do assoalho pélvico (AFA), cone vaginal, ultrassonografia, ressonância magnética e palpação digital da musculatura vaginal (Andrade, 2021) (Figura 4).

O método de eletromiografia (EMG) trás a aquisição de parâmetros eletrofisiológicos que definem o músculo estriado esquelético constituindo normal ou anormal (Lose et al., 1998). A EMG registra as potencialidades de elétricos gerados pelo despolarização de fibras musculares em repouso e durante a contração voluntária; avalia a capacidade de contração e registra a sua amplitude em microvolts $(\mu \mathrm{V})$ (Rett et al., 2005). 
Figura 4. Dinamômetro vaginal, eletromiógrafo e perineômetro, respectivamente.

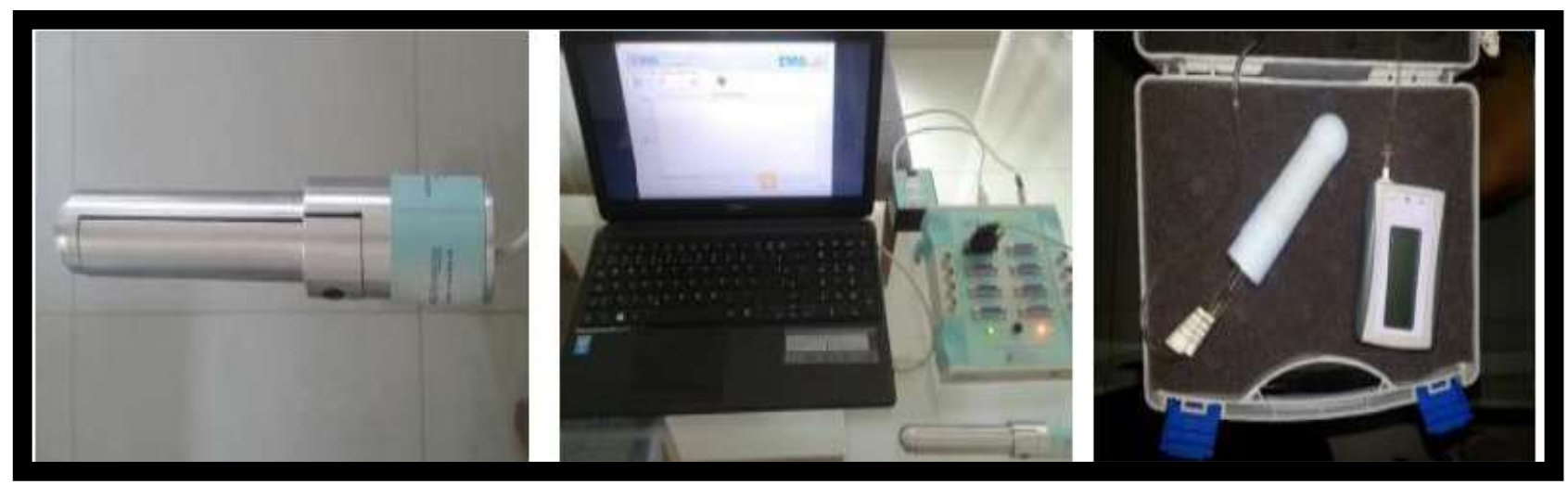

Fonte: adaptado de https://repositorio.animaeducacao.com.br/handle/anima/13856

Diversas são as vertentes que envolvem a fisioterapêutica do fortalecimento pélvico pós-parto normal (Andrade, 2021; Assis, 2010; Berghmans, 2018; de Castro Cruz et al., 2019; Pedraza et al., 2014, 2018; Vural, 2018) e há casos de reabilitação pós parto utilizando a fisioterapia e atividade física na sua recuperação (Vural, 2018). A frequência de exercícios é de três séries de dez repetições durante duas a três vezes na semana (de Castro Cruz et al., 2019) utilizando exercícios de fortalecimento por meio de programas como Pilates, métodos utilizando bolas ginásticas e também aparelhos de estimulação (Andrade, 2021; de Castro Cruz et al., 2019; Pedraza et aL., 2018). O nascimento do bebê e durante a gestação levam à esforços musculares importantes e posteriormente, sua respectiva fraqueza, a qual se não tratada leva a condições de incontinência urinária (Nygaard et al., 2017). Nolasco et al. (2007), em seu estudo, enfatizou que uma ferramenta chave para ser associada no tratamento fisioterápico para o fortalecimento dos músculos do assoalho pélvico, trabalhando de forma ativa conforme orientada como as técnicas de cinesioterapia mais utilizada na reeducação perineal, e considerado os exercícios de kegel, originado pelo ginecologista Arnold kegel em 1950, que desenvolveu um programa de exercícios de fortalecimento para o MAP (Nolasco, et. al, 2008).

O autoconhecimento do fortalecimento do assoalho pélvico são alguns recursos que pode se utilizar para o tratamento de disfunções sexuais, aumentando o desejo sexual e a excitação. Através de exercícios ou alguns recursos da eletroterapia aonde contribuem para fortalecimento, além disso os músculos que compõe o corpo cavernoso do clitóris têm uma melhor resposta sensório-motora quando se realiza contração involuntária dos músculos do assoalho pélvico durante o orgasmo além de tudo e importante enfatizar que os exercícios trás uma melhora na sexualidade, são exercícios terapêuticos que prevenir patologias como prolapso genital, disfunções sexuais e incontinência urinária (Nagamine et al., 2021)

Existe uma grande importância da fisioterapêutica no tratamento de enfermidades causadas após o parto normal, uma vez que há grande dilatação do aparelho do assoalho pélvico e sua respectiva musculatura (Uldahl et al., 2021; Vural, 2018).

\section{Conclusão}

Com base nestas informações, foram amplamente trazidos neste trabalho informações acerca da anatomia correspondente ao assoalho pélvico, comumente afetado em situações de partos como parto normal, o qual ocorre uma grande dilatação óssea e muscular. Além disso, foram apresentadas informações acerca das enfermidades causadas pela fraqueza muscular e suas vertentes. Um programa fisioterapêutico nestas situações é de suma importância para qualidade de vida da mulher e associados. 


\section{Referências}

Anastasiadi, Z., Lianos, G. D., Ignatiadou, E., Harissis, H. V., \& Mitsis, M. (2017). Breast cancer in young women: an overview. In Updates in Surgery.

Andrade, B. C. D. E. (2021). importância do fortalecimento do assoalho pélvico em gestantes. UniAGES Centro Universitário Bacharelado em Fisioterapia.

Assis, L. C. de. (2010). Efetividade de exercícios do assoalho pélvico durante a gestação como medida preventiva da incontinência urinária e da disfunção muscular do assoalho pélvico - Dissertação (mestrado). Dissertação (mestrado), 01 of 90.

Berghmans, B. (2018). Physiotherapy for pelvic pain and female sexual dysfunction: an untapped resource. International Urogynecology Journal, 29(5), 631638.

Nagamine, B. P. ., Dantas, R. da S. ., \& Silva, K. C. C. da . (2021). The importance of strengthening the pelvic floor muscles in women's health. Research, Society and Development, 10(2), e56710212894. https://doi.org/10.33448/rsd-v10i2.12894

Castro LA, Machado GC, Trindade 2019;56(S4):39-51APNT. Fisioterapia em mulheres com incontinência urinária-relatos de caso. Revista UNINGÁ.

De Castro Cruz, A., Fonseca, S. T., Araújo, V. L., Da Silva Carvalho, D., Barsante, L. D., Pinto, V. A., \& Souza, T. R. (2019). Pelvic drop changes due to proximal muscle strengthening depend on foot-ankle varus alignment. Applied Bionics and Biomechanics, 2019. https://doi.org/10.1155/2019/2018059

Goodrich, F. W. (1953). The theory and practice of natural childbirth. The Yale journal of biology and medicine, 25(6), 529-534.

Lose, G., Fantl, J. A., Victor, A., Walter, S., Wells, T. L., Wyman, J., \& Mattiasson, A. (2001). Outcome measures for research in adult women with symptoms of lower urinary tract dysfunction. Standardization Committee of the International Continence Society. Acta obstetricia et gynecologica Scandinavica, 80(11), 981-985. https://doi.org/10.1034/j.1600-0412.2001.801102.x

Moreno AL. Fisioterapia em uroginecologia. 2009. (2 ed.) Barueri: Manole;

Netter. (2000). Atlas de Anatomia Humana (Novartis (org.); (2 $2^{\circ}$ ed).

Nolasco, J.; Martins, Letícia Berquo, M.; Alves Sandoval, R. (2008). Atuação da cinesioterapia no fortalecimento muscular do assoalho pélvico feminino: revisão bibliográfica. EFDeportes.com, Revista Digital, 12(117), 1-8.

Nygaard IE, Clark E, Clark L, et al Physical and cultural determinants of postpartum pelvic floor support and symptoms following vaginal delivery: a protocol for a mixed-methods prospective cohort study BMJ Open 2017;7:e014252. doi: 10.1136/bmjopen-2016-014252

Pereira A. S. et al. (2018). Metodologia da pesquisa científica. UFSM

Pedraza, R., Nieto, J., Ibarra, S., \& Haas, E. M. (2014). Pelvic muscle rehabilitation: A standardized protocol for pelvic floor dysfunction. Advances in Urology, 2014, 1-7. https://doi.org/10.1155/2014/487436

R. Pedraza et al., "Efetividade de exercícios do assoalho pélvico durante a gestação como medida preventiva da incontinência urinária e da disfunção muscular do assoalho pélvico - Dissertação (mestrado)", Adv. Urol., 64(2), 01 of 90, 2018, doi: 10.1007/s00192-017-3536-8.

Permanyer, I., \& Boertien, D. (2019). A century of change in global education variability and gender differences in education. PLoS ONE, 14(2), 1-22. https://doi.org/10.1371/journal.pone.0212692

Rett, M.T.; et al. Existe diferença na contratilidade da musculature do assoalho pélvico feminino em diversas posições? Ver brasileira ginecol obstet.,v. 27, p. 20- 23, 2005. RETT, M.T.; et al. Existe diferença na contratilidade da musculature do assoalho pélvico feminino em diversas posições? Ver brasileira ginecol obstet., 27, 20-23, 2005.

Uldahl, M., Christensen, J. W., \& Clayton, H. M. (2021). Relationships between the Rider's pelvic mobility and balance on a gymnastic ball with equestrian skills and effects on horse welfare. Animals, 11(2), 1-14. https://doi.org/10.3390/ani11020453

Vural, M. (2018). Pelvic pain rehabilitation. Turkish Journal of Physical Medicine and Rehabilitation, 64(4), 291-299. https://doi.org/10.5606/tftrd.2018.3616 